\title{
Single top squark production as a probe of natural supersymmetry at the LHC
}

\author{
Ken-ichi Hikasa, ${ }^{1, *}$ Jinmian $\mathrm{Li}^{,}{ }^{2}, \dagger$ Lei $\mathrm{Wu},{ }^{3, \ddagger}$ and Jin Min Yang ${ }^{4, \S}$ \\ ${ }^{1}$ Department of Physics, Tohoku University, Sendai 980-8578, Japan \\ ${ }^{2} A R C$ Centre of Excellence for Particle Physics at the Terascale, \\ Department of Physics, University of Adelaide, Adelaide, SA 5005, Australia \\ ${ }^{3}$ ARC Centre of Excellence for Particle Physics at the Terascale, \\ School of Physics, The University of Sydney, NSW 2006, Australia \\ ${ }^{4}$ State Key Laboratory of Theoretical Physics, Institute of Theoretical Physics, Academia Sinica, Beijing 100190, China
}

(Dated: October 10, 2018)

\begin{abstract}
Light top squarks (stops) and light higgsinos are the key features of natural SUSY, where the higgsinos $\tilde{\chi}_{1}^{ \pm}$and $\tilde{\chi}_{1,2}^{0}$ are nearly degenerate and act as the missing transverse energy $\left(\mathbb{E}_{T}\right)$ at the LHC. Besides the strong production, the stop can be produced via the electroweak interaction. The determination of the electroweak properties of the stop is an essential task for the LHC and future colliders. So in this paper, we investigate the single stop $\left(\tilde{t}_{1}\right)$ production $p p \rightarrow \tilde{t}_{1}+\mathbb{E}_{T}$ in the natural SUSY at the LHC, which gives the monotop signature $t+\mathbb{E}_{T}$ from $\tilde{t}_{1} \rightarrow t \tilde{\chi}_{1,2}^{0}$ or the monobottom signature $b+\mathbb{E}_{T}$ from $\tilde{t}_{1} \rightarrow b \tilde{\chi}_{1}^{+}$. We perform Monte Carlo simulations for these signatures and obtain the results: (1) The signal $b+\mathbb{E}_{T}$ has a better sensitivity than $t+\mathbb{E}_{T}$ for probing natural SUSY; (2) The parameter region with a higgsino mass $100 \mathrm{GeV} \lesssim \mu \lesssim 225 \mathrm{GeV}$ and stop mass $m_{\tilde{t}_{1}} \lesssim$ $620 \mathrm{GeV}$, can be probed through the single stop production with $S / \sqrt{B}>3$ and $4 \% \lesssim S / B \lesssim 19 \%$ at $14 \mathrm{TeV}$ HL-LHC with an integrated luminosity of $3000 \mathrm{fb}^{-1}$.
\end{abstract}

\section{INTRODUCTION}

The search for supersymmetry (SUSY) is a longstanding important task in particle physics. One prime motivation for weak-scale SUSY is that it protects the Higgs vacuum expectation value without unnatural finetuning of the theory parameters. In the minimal supersymmetric standard model (MSSM), only a small subset of the supersymmetric partners strongly relates with the naturalness of the Higgs potential [1]. This can be seen from the minimization of the Higgs potential [2]:

$$
\begin{aligned}
\frac{M_{Z}^{2}}{2} & =\frac{\left(m_{H_{d}}^{2}+\Sigma_{d}\right)-\left(m_{H_{u}}^{2}+\Sigma_{u}\right) \tan ^{2} \beta}{\tan ^{2} \beta-1}-\mu^{2} \\
& \simeq-\mu^{2}-\left(m_{H_{u}}^{2}+\Sigma_{u}\right),
\end{aligned}
$$

where $\mu$ is the higgsino mass parameter, and $m_{H_{d}}^{2}$ and $m_{H_{u}}^{2}$ denote the weak scale soft SUSY breaking masses of the Higgs fields. A moderate or large $\tan \beta \equiv v_{u} / v_{d}$ is assumed in the last approximate equality. $\Sigma_{u}$ and $\Sigma_{d}$ arise from the radiative corrections to the Higgs potential, and the one-loop dominant contribution to $\Sigma_{u}$ is given by [3]

$$
\Sigma_{u} \sim \frac{3 Y_{t}^{2}}{16 \pi^{2}} \times m_{\tilde{t}_{i}}^{2}\left(\log \frac{m_{\tilde{t}_{i}}^{2}}{Q^{2}}-1\right) .
$$

In order to obtain the observed value of $M_{Z}$ without large cancelations in Eq. (1), each term on the right hand side should be comparable in magnitude. Thus, the higgsino mass $\mu$ must be of the order of $\sim 100-200 \mathrm{GeV}$ and the requirement of $\Sigma_{u} \sim M_{Z}^{2} / 2$ produces an upper bound on the stop mass, which is about $500 \mathrm{GeV}[4,5]$ (a 125 $\mathrm{GeV}$ Higgs mass can be achieved by a large stop trilinear coupling without very heavy stops in the MSSM or achieved by extending the MSSM with additional Dterms or F-terms [6-8]). In addition, since the gluino contributes to $m_{H_{u}}$ at two-loop level, it is also upper bounded by the naturalness [9] (however, the direct LHC searches have pushed the gluino up to TeV scale $[10,11]$ while the recent ATLAS Z-peaked excess may indicate a gluino around $800 \mathrm{GeV}[12])$.

So to test SUSY naturalness, the crucial task is to search for light stops or higgsinos. The search strategy for the pair productions of these nearly degenerate higgsinos has been recently studied [13-26]. During the LHC run-1, the ATLAS and CMS collaborations have performed the extensive searches for the stops through the gluino-mediated stop production $[27,28]$ or the direct stop pair production $[29,30]$. Meanwhile, many theoretical studies that aim for improving the LHC sensitivity to a light stop have been proposed [31-46]. The current LHC constraints indicate a stop mass bound of hundreds of $\mathrm{GeV}$ [47-57], however, those results are affected by the stop polarization states and branching ratios. The constraints on the right-handed stop from the LHC run1 direct searches [29,30] are usually weakened by the branching ratio suppression, which can still be as light as $230 \mathrm{GeV}$ in some compressed region [58]. If the stop mass is heavier than about $450 \mathrm{GeV}$, it is allowed in most parameter space. So in our work, we require the stop mass be heavier than $450 \mathrm{GeV}$ [58].

Usually, the stop pair production provides the most sensitive way to search for the stop at the LHC. However, the stop can also participate in the electroweak interaction processes. The determination of the electroweak properties of the stop is an essential task for the LHC and future colliders. In this work, we study the single stop production $p p \rightarrow \tilde{t}_{1}+\mathbb{E}_{T}$ in the natural SUSY at 
the LHC. The observation of the single stop production will test the electroweak properties of the stop and the naturalness of the supersymmetry. In the following, we will perform the Monte Carlo simulations for the single stop production and examine its sensitivity at the LHC.

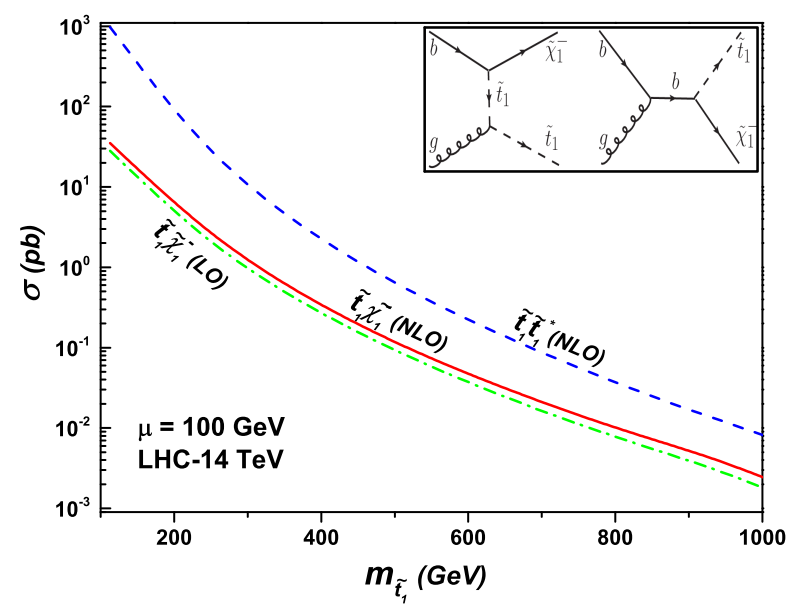

Figure 1: The cross sections of $\tilde{t}_{1} \tilde{t}_{1}^{*}$ and $\tilde{t}_{1} \tilde{\chi}_{1}^{-}$productions at the $14 \mathrm{TeV}$ LHC for $\tan \beta=50$ and degenerate higgsinos with mass $\mu=100 \mathrm{GeV}$. The contribution of conjugate process $\tilde{t}_{1}^{*} \tilde{\chi}_{1}^{+}$is included.

\section{CALCULATIONS AND SIMULATIONS}

At the LHC, the single stop production is induced by the electroweak interaction and proceeds through the following process (see Fig. 1 for the corresponding Feynman diagrams):

$$
p p \rightarrow \tilde{t}_{1} \tilde{\chi}_{1}^{-} .
$$

Since in natural SUSY the light higgsinos are nearly degenerate, the decay products of $\tilde{\chi}_{1}^{-} \rightarrow W^{*} \chi_{1}^{0}$ will carry small energies and, hence, are too soft to be observed in the detector. Thus, the associated production of $\tilde{t}_{1} \tilde{\chi}_{1}^{-}$ can be identified as $\tilde{t}_{1}+\mathbb{E}_{T}$, which provides a distinctive signature at the LHC.

In Fig. 1, we show the next-to-leading order (NLO) cross sections of the stop pair and the single stop productions for $\mu=100 \mathrm{GeV}$ at $14 \mathrm{TeV}$ LHC by using the packages Prospino2 [59] and MadGolem [60], respectively. The renormalization and factorization scales are taken as the half average of the final states masses. In the calculations of $\tilde{t}_{1} \tilde{\chi}_{1}^{-}$, we use the LO and NLO parton densities given by CTEQ6L1 and CTEQ6M with five active flavors [61]. The contribution of the conjugate process $\tilde{t}_{1}^{*} \tilde{\chi}_{1}^{+}$is included. Except for the higgsino mass parameter $\mu$ and right-handed stop soft mass $m_{U_{3}}$, we assume other soft supersymmetric masses at $1 \mathrm{TeV}$, and use the packages SOFTSUSY-3.3.9 [62] and MSSMCalc [63] to calculate masses, couplings and branching ratios of the sparticles.
Since the cross section of single stop production is not sensitive to $\tan \beta$, we take $\tan \beta=50$ for simplicity. We find that the single stop cross section can still reach about $200 \mathrm{fb}$ when $m_{\tilde{t}_{1}} \simeq 450 \mathrm{GeV}$. The NLO $K$-factor of the process $p p \rightarrow \tilde{t}_{1} \tilde{\chi}_{1}^{-}$ranges from 1.25 to 1.33 . When the stop becomes heavy, the single stop production cross section will decrease, but slower than the pair production, due to the kinematics.

Next, we investigate the LHC observability of the single stop signatures with the sequent decays $\tilde{t}_{1} \rightarrow t \tilde{\chi}_{1,2}^{0}$ and $\tilde{t}_{1} \rightarrow b \tilde{\chi}_{1}^{+}$:

$$
\begin{aligned}
& p p \rightarrow \tilde{t}_{1} \tilde{\chi}_{1}^{-} \rightarrow t \tilde{\chi}_{1,2}^{0} \tilde{\chi}_{1}^{-} \rightarrow b j j+\mathbb{E}_{T}, \\
& p p \rightarrow \tilde{t}_{1} \tilde{\chi}_{1}^{-} \rightarrow b \tilde{\chi}_{1}^{+} \tilde{\chi}_{1}^{-} \rightarrow b+\mathbb{E}_{T} .
\end{aligned}
$$

For the decay $\tilde{t}_{1} \rightarrow t \tilde{\chi}_{1,2}^{0}$, the SM backgrounds to the signal $b j j+\mathbb{E}_{T}$ are from the semi- and full-hadronic $t \bar{t}$ events $[64-66]^{1}$, where the undetected lepton and the limited jet energy resolution will lead to the relatively large missing transverse energy. The processes $W+$ jets and $Z+$ jets can fake the signal when one of those light-flavor jets are mis-tagged as a $b$-jet. Also, the single top can mimic our signal when the lepton from the $W$ boson decay is missed at the detector. While $t \bar{t}+V$ backgrounds are not considered in our simulations due to their small missing energy or cross sections compared to the above backgrounds.

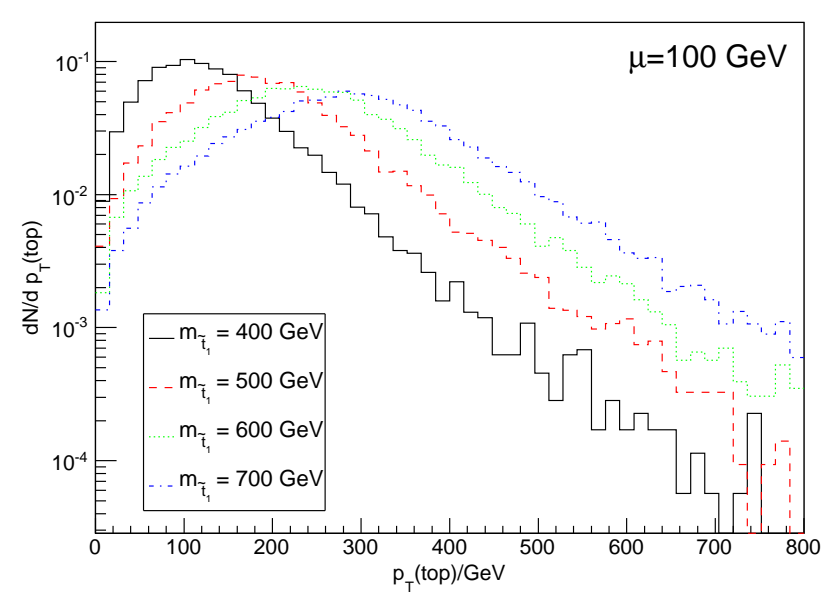

Figure 2: The parton-level $p_{T}$ distribution of the top quark in the channel $\tilde{t}_{1} \rightarrow t \tilde{\chi}_{1,2}^{0}$ for $\mu=100 \mathrm{GeV}$ at $14 \mathrm{TeV}$ LHC.

\footnotetext{
${ }^{1}$ Hadronic monotop has received special attention since its signature offers the possibility to use top reconstruction as a tool to reject the backgrounds. In contrast, the leptonic monotops is believed to be more challenging since the branching fraction of the leptonic top quark decay is smaller and since there are two different sources of missing transverse energy, namely a neutrino coming from the top quark decay and the new invisible state. In Ref.[65], the authors comparatively studies these two channels and found that the sensitivity of both channels are very similar.
} 
In Fig. 2, we present the parton-level $p_{T}$ distribution of the top quark in the channel $\tilde{t}_{1} \rightarrow t \tilde{\chi}_{1,2}^{0}$ for $\mu=100$ $\mathrm{GeV}$ at $14 \mathrm{TeV} \mathrm{LHC}$. It can be seen that, with the increase of stop mass, the top quark produced from stop decay is boosted and has larger $p_{T}$. So, in the analysis of $\tilde{t}_{1} \rightarrow t \tilde{\chi}_{1,2}^{0}$ channel, we respectively adopt HEPTopTagger [32] and normal hadronic top reconstruction methods for each sample to identify the top quark in the final states and present our results with the best one. The detailed analysis strategies are the followings:

- Events with any isolated leptons are rejected;

- Method-1: We use Cambridge-Aachen (CA) algorithms [67] in Fastjet [68] to cluster the jets with $R=1.5$ to obtain the top-jet candidates. Each candidate must have the top quark substructure required by the HEPTopTagger. The $b$-tagging is also imposed in the top-jet reconstruction. Other energy deposits outside the top-jet are further reconstructed as the normal jets by using anti- $k_{t}$ algorithm with $R=0.4$ [69]. The top window used in our analysis is $150<m_{t}<200 \mathrm{GeV}$. While the $W$ window is taken as the default value in HepTopTagger;

- Method-2: In normal hadronic top quark reconstruction, a pair of jets is selected with the invariant mass $m_{j j}>60 \mathrm{GeV}$ and the smallest $\Delta R$. A third jet closest to this di-jet system is used to constitute the top quark candidate. Among these three jets, at least one $b$-jet and $\Delta \phi\left(\mathbb{E}_{T}, p_{T}\left(b_{1}\right)\right)>1$ is required. The anti- $k_{t}$ algorithm is used for jet clustering with $R=0.4$;

- We keep the events with the exact one reconstructed top quark and require $150 \mathrm{GeV}<m_{t}^{\text {rec }}<$ $200 \mathrm{GeV}$;

- The extra leading jet $j_{1}$ outside the reconstructed top quark object is vetoed if $p_{T}\left(j_{1}\right)>30 \mathrm{GeV}$ and $\left|\eta\left(j_{1}\right)\right|<2.5$

- We define the signal regions according to $\left(\mathbb{E}_{T}, p_{T}\left(j_{\text {top }}\right)\right)$ cuts: $(200,100),(250,150),(300$, $200),(350,250)$ for Method-1, and $\left(p_{T}(b), \mathbb{E}_{T}\right)$ cuts: $(200,50),(250,50),(300,100),(350,100)$ GeV for Method-2.

For the decay $\tilde{t}_{1} \rightarrow b \tilde{\chi}_{1}^{+}$, the SM backgrounds to the signal $b+\mathbb{E}_{T}$ are dominated by the processes $W+$ jets and $Z+$ jets when the light-flavor jets are mis-identified as $b$-jets [70]. The $t \bar{t}$ events become the sub-leading backgrounds due to their large multiplicity. The signal events are selected to satisfy the following criteria:

- Events with any isolated leptons are rejected;
- We require exact one hard $b$-jet in the final states, but allow an additional softer jet with $p_{T}\left(j_{1}\right)<30$ $\mathrm{GeV}$ and $\Delta \phi\left(\mathbb{E}_{T}, p_{T}\left(j_{1}\right)\right)>2$. Since the hardness of $b$-jet from stop decay depends on the mass splitting between $\tilde{t}_{1}$ and $\tilde{\chi}_{1}^{-}$, we define three signal regions for each sample according to $\left(\mathbb{E}_{T}, p_{T}(b)\right)$ cuts: $(100,70),(150,100)$ and $(250,200) \mathrm{GeV}$.

Finally, we use the most sensitive signal region (with the highest $S / \sqrt{B}$ ) for each decay mode and show our results in Fig. 3 and Fig. 4, respectively. In our study, we omitted the QCD multijet backgrounds, whose correct treatment needs the experimental data-driven methods and hence depends on the realistic detector environments of the $14 \mathrm{TeV}$ LHC. As discussed in [65, 70, 71], the requirements of high $p_{T}\left(b_{1}\right)$ and large $\mathbb{E}_{T}$ with a seperation $\Delta \phi\left(\mathbb{E}_{T}, p_{T}\left(j_{1}\right)\right)$ can usually be expected to greatly reduce the fake contamination from the QCD backgrounds and allow for a good signal selection efficiency.

The parton level signal and background events are generated with MadGraph5 [72], where $W / Z+$ jets is matched up to 3 jets by using MLM matching scheme [73] and setting $x q c u t=30 \mathrm{GeV}$. For the value of qcut in matching, we take it to $\max (x q c u t+5, x q c u t * 1.2)[74]$ in our simulation. We perform parton shower and fast detector simulations with PYTHIA [75] and Delphes [76]. We assume the $b$-jet tagging efficiency as 70\% [77] and a misidentification efficiency of $c$-jets and light jets as $10 \%$ and $0.1 \%$, respectively. The cross section of $t \bar{t}$ is normalized to the approximately next-to-next-to-leading order value $\sigma_{t \bar{t}}=920 \mathrm{pb}[78]$.

\section{RESULTS AND DISCUSSIONS}

Table I: The cross sections of $V+$ jets, $t \bar{t}$ and $\tilde{t}_{1}\left(\rightarrow t \tilde{\chi}_{1,2}^{0}\right) \tilde{\chi}_{1}^{-}$for a benchmark point $\left(m_{\tilde{t}_{1}}, \mu\right)=(611,100) \mathrm{GeV}$ and $\tan \beta=10$ in Method-1 and Method-2 at $14 \mathrm{TeV}$ LHC with $\mathcal{L}=3000$ $\mathrm{fb}^{-1}$. The cross sections are in unit of $\mathrm{fb}$.

\begin{tabular}{|c|c|c|c|c|c|c|c|}
\hline cuts & $W+$ jets & $Z+$ jets & $t \bar{t}$ & $t W$ & $S$ & $S / B$ & $S / \sqrt{B}$ \\
\hline Method-1 & $<10^{-2}$ & 0.29 & 2.20 & 0.80 & 0.13 & $4.0 \%$ & 3.9 \\
\hline Method-2 & $<10^{-2}$ & 0.59 & 0.55 & 0.24 & 0.044 & $3.2 \%$ & 2.1 \\
\hline
\end{tabular}

In Table I, we compare the results of $p p \rightarrow \tilde{t}_{1}(\rightarrow$ $\left.t \tilde{\chi}_{1,2}^{0}\right) \tilde{\chi}_{1}^{-}$for a benchmark point $\left(m_{\tilde{t}_{1}}, \mu\right)=(611,100)$ GeV in Method-1 and Method-2 at 14 TeV LHC. From this table we can see that the $Z+$ jets background in Method-1 is smaller than in Method-2, while the $t \bar{t}$ background in Method-1 is larger than in Method-2. However, the signal events can be more kept in Method-1 than in Method-2. So the overall effects make the Method-1 have a better sensitivity in reconstructing the top quark in the region with large mass splitting between $\tilde{t}_{1}$ and $\tilde{\chi}_{1}^{-}$. At $14 \mathrm{TeV}$ LHC with $\mathcal{L}=3000 \mathrm{fb}^{-1}$, the statistical signif- 
icance $S / \sqrt{B}$ for our benchmark point can reach $3.9 \sigma$ $(2.1 \sigma)$ with $S / B=4.0 \%(3.2 \%)$ in Method-1(2).

Table II: The cross sections of $V+$ jets, $t \bar{t}$ and $\tilde{t}_{1}\left(\rightarrow b \tilde{\chi}_{1}^{+}\right) \tilde{\chi}_{1}^{-}$for a benchmark point $\left(m_{\tilde{t}_{1}}, \mu\right)=(496,200) \mathrm{GeV}$ and $\tan \beta=10$ at $14 \mathrm{TeV}$ LHC with $\mathcal{L}=3000 \mathrm{fb}^{-1}$. The cross sections are in unit of $\mathrm{fb}$.

\begin{tabular}{|c|c|c|c|c|c|}
\hline$W+$ jets & $Z+$ jets & $t \bar{t}$ & $S$ & $S / B$ & $S / \sqrt{B}$ \\
\hline$<10^{-2}$ & 2.77 & 1.10 & 0.20 & $5.1 \%$ & 5.5 \\
\hline
\end{tabular}

In Table II, we show the cross sections of $V+$ jets, $t \bar{t}$ and $\tilde{t}_{1}\left(\rightarrow b \tilde{\chi}_{1}^{+}\right) \tilde{\chi}_{1}^{-}$for a benchmark point $\left(m_{\tilde{t}_{1}}, \mu\right)=$ $(496,200) \mathrm{GeV}$ at the $14 \mathrm{TeV}$ LHC. Different from $\tilde{t}_{1} \rightarrow$ $t \tilde{\chi}_{1,2}^{0}$ channel, $Z+$ jets background is dominant over $t \bar{t}$ since only one hard $b$-jet is required in the final state. From Table II we can see that $S / \sqrt{B}$ and $S / B$ can reach 5.5 and $5.1 \%$ for $\mathcal{L}=3000 \mathrm{fb}^{-1}$, respectively.

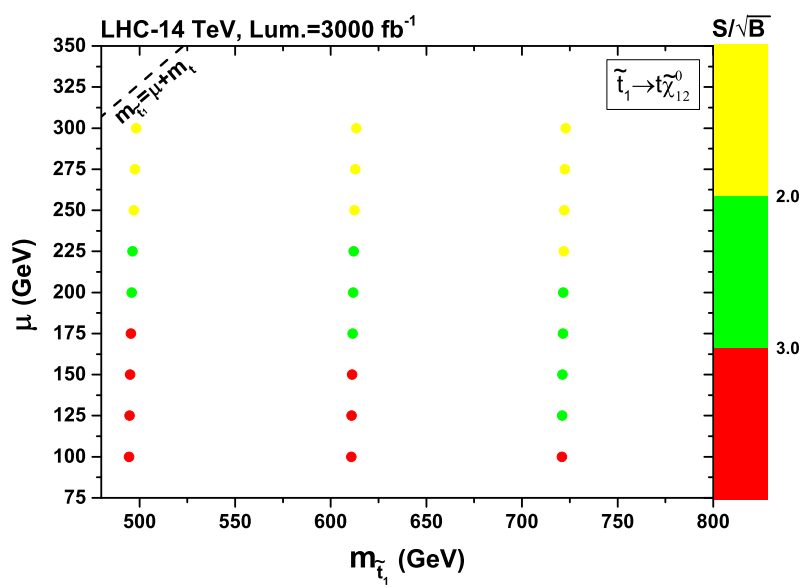

Figure 3: The dependence of the significance of the channel $\tilde{t}_{1} \rightarrow t \tilde{\chi}_{1,2}^{0}$ on the higgsino mass $\mu$ and stop mass $m_{\tilde{t}_{1}}$ at the $14 \mathrm{TeV}$ LHC with $\mathcal{L}=3000 \mathrm{fb}^{-1}$.

In Fig. 3, we display the dependence of statistical significance $S / \sqrt{B}$ of the channel $\tilde{t}_{1} \rightarrow t \tilde{\chi}_{1,2}^{0}$ on the higgsino mass $\mu$ and stop mass $m_{\tilde{t}_{1}}$ at $14 \mathrm{TeV}$ LHC with $\mathcal{L}=3000$ $\mathrm{fb}^{-1}$. We can see that values of $S / \sqrt{B}$ decrease with the increase of $\mu$ because of the cut efficiency reduction. When the stop becomes heavy, the cross section of $\tilde{t}_{1} \tilde{\chi}_{1}^{-}$ is suppressed. However, as a result of the application of HEPTopTagger method, more signal events can be kept, in particular in the mass range $450 \mathrm{GeV} \lesssim m_{\tilde{t}_{1}} \lesssim 650$ $\mathrm{GeV}$. Therefore, when $\mu \lesssim 150 \mathrm{GeV}$, the stop mass $m_{\tilde{t}_{1}} \lesssim$ $610 \mathrm{GeV}$ can be probed at $\gtrsim 3 \sigma$ statistical significance with $S / B \lesssim 8 \%$.

In Fig. 4 , the statistical significance $S / \sqrt{B}$ of the channel $\tilde{t}_{1} \rightarrow b \tilde{\chi}_{1}^{+}$is presented on the plane of higgsino mass $\mu$ versus stop mass $m_{\tilde{t}_{1}}$ at $14 \mathrm{TeV}$ LHC with $\mathcal{L}=3000$ $\mathrm{fb}^{-1}$. It can be seen that the sensitive stop region lies in $450 \mathrm{GeV} \lesssim m_{\tilde{t}_{1}} \lesssim 620 \mathrm{GeV}$, where a hard $b$-jet $\left(p_{T}>200\right.$ $\mathrm{GeV})$ and the sizable $\mathbb{E}_{T}\left(\mathbb{E}_{T}>250 \mathrm{GeV}\right)$ can be used to

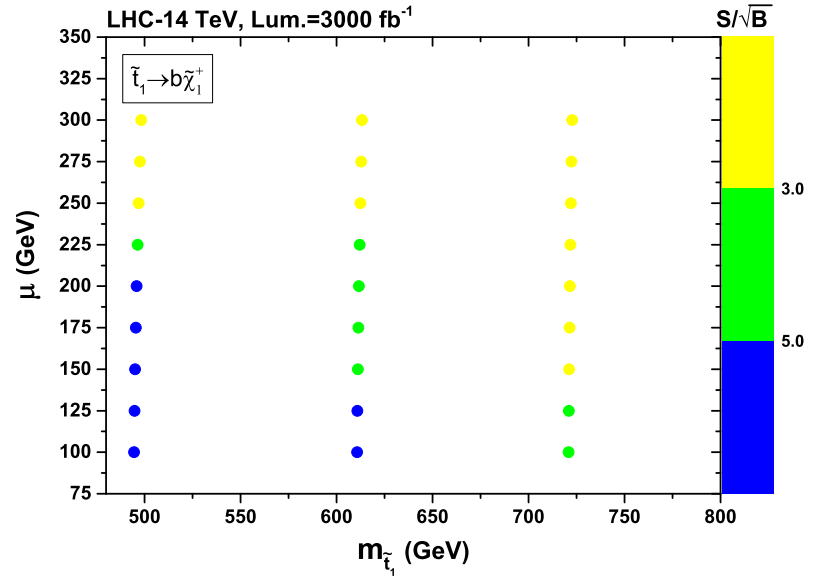

Figure 4: Same as Fig. 3, but for the decay channel $\tilde{t}_{1} \rightarrow b \tilde{\chi}_{1}^{+}$.

effectively suppress the backgrounds. But when the stop mass increases, $S / \sqrt{B}$ will rapidly decrease. We see that the higgsino mass $100 \mathrm{GeV} \lesssim \mu \lesssim 225 \mathrm{GeV}$ and the stop mass $m_{\tilde{t}_{1}} \lesssim 620 \mathrm{GeV}$ can be covered at $\gtrsim 3 \sigma$ statistical significance with $S / B$ varying from $4 \%$ to $19 \%$.

\section{CONCLUSIONS}

even its exclusion limit for the stop may be not as good as the pair production. However, the stop can participates in the strong interaction processes but also in the electroweak interaction processes, the determination of the electroweak properties of the stop is an essential task for the LHC and future colliders. In this work we propose to probe natural SUSY by using the electroweak single-stop production $p p \rightarrow \tilde{t}_{1}+\mathbb{E}_{T}$ at the LHC (here the missing energy is from the nearly degenerate higgsinos). By analyzing the decay channels of the stop $\tilde{t}_{1} \rightarrow t \tilde{\chi}_{1,2}^{0}$ and $\tilde{t}_{1} \rightarrow b \tilde{\chi}_{1}^{+}$, we obtain the obervations: (1) The decay $\tilde{t}_{1} \rightarrow b \tilde{\chi}_{1}^{+}$has a better sensitivity than $\tilde{t}_{1} \rightarrow t \tilde{\chi}_{1,2}^{0}$ ; (2) The parameter region with a higgsino mass 100 $\mathrm{GeV} \lesssim \mu \lesssim 225 \mathrm{GeV}$ and the stop mass $m_{\tilde{t}_{1}} \lesssim 620 \mathrm{GeV}$ can be covered with $S / \sqrt{B}>3$ and $4 \% \lesssim S / B \lesssim 19 \%$ at $14 \mathrm{TeV}$ HL-LHC with an integrated luminosity of 3000 $\mathrm{fb}^{-1}$. So the searches for the single stop production will directly test the naturalness of the supersymmetry and the electroweak properties of the stop.

\section{ACKNOWLEDGMENTS}

Lei Wu thanks David Lopez-Val and Dorival Goncalves for providing us the MadGolem package. This work was partly supported by the Grant-in-Aid for Scientific Research (No. 24540246) from Ministry of Education, Culture, Sports, Science and Technology (MEXT) of Japan, by the Australian Research Council, by the CAS Cen- 
ter for Excellence in Particle Physics (CCEPP), by the National Natural Science Foundation of China (NNSFC) under grants Nos. 11305049, 11275057, 11375001, 11405047, 11275245, 10821504 and 11135003, and by Specialized Research Fund for the Doctoral Program of Higher Education under Grant No.20134104120002.

* Electronic address:hikasa@phys.tohoku.ac.jp

$\dagger$ Electronic address:jinmian.li@adelaide.edu.au

¥ Electronic address:leiwu@physics.usyd.edu.au

$\S$ Electronic address:jmyang@itp.ac.cn

[1] R. Barbieri and G. F. Giudice, Nucl. Phys. B 306, 63 (1988).

[2] R. Arnowitt and P. Nath, Phys. Rev. D 46, 3981 (1992).

[3] H. Baer, et al., Phys. Rev. D 87, 115028 (2013).

[4] S. F. King, M. Muhlleitner and R. Nevzorov, Nucl. Phys. B 860, 207 (2012).

[5] Z. Kang, J. Li and T. Li, JHEP 1211, 024 (2012).

[6] T. Han, P. Langacker and B. McElrath, Phys. Rev. D 70, 115006 (2004).

[7] Y. Zhang, H. An, X. d. Ji and R. N. Mohapatra, Phys. Rev. D 78, 011302 (2008).

[8] J. Cao et al., JHEP 1203, 086 (2012) [arXiv:1202.5821 [hep-ph]].

[9] C. Brust, A. Katz, S. Lawrence and R. Sundrum, JHEP 1203, 103 (2012).

[10] G. Aad et al. [ATLAS Collaboration], JHEP 1310, 130 (2013); S. Chatrchyan et al. [CMS Collaboration], JHEP 1406, 055 (2014).

[11] T. Cheng, J. Li, T. Li and Q. S. Yan, Phys. Rev. D 89, 015015 (2014).

[12] G. Aad et al. [ATLAS Collaboration], arXiv:1503.03290 [hep-ex]; G. Barenboim et al., arXiv:1503.04184 [hep-ph]; U. Ellwanger, arXiv:1504.02244 [hep-ph]; B. Allanach, A. Raklev and A. Kvellestad, arXiv:1504.02752 [hepph]; A. Kobakhidze, N. Liu, L. Wu and J. M. Yang, arXiv:1504.04390 [hep-ph]; J. Cao, L. Shang, J. M. Yang and Y. Zhang, arXiv:1504.07869 [hep-ph].

[13] G. F. Giudice and A. Pomarol, Phys. Lett. B 372, 253 (1996)

[14] K. L. Chan, U. Chattopadhyay and P. Nath, Phys. Rev. D 58, 096004 (1998).

[15] S. P. Martin, Phys. Rev. D 89, 035011 (2014).

[16] H. Baer, et al., Phys. Rev. Lett. 109, 161802 (2012).

[17] I. Gogoladze, F. .Nasir and Q. .Shafi, Int. J. Mod. Phys. A 28, 1350046 (2013).

[18] S. Gori, S. Jung and L. T. Wang, JHEP 1310, 191 (2013).

[19] H. Baer, et al., Phys. Rev. Lett. 110, 151801 (2013).

[20] C. Han, et al., JHEP 1402, 049 (2014).

[21] P. Schwaller and J. Zurita, JHEP 1403, 060 (2014).

[22] Z. Han, G. D. Kribs, A. Martin and A. Menon, Phys. Rev. D 89, 075007 (2014).

[23] H. Baer, A. Mustafayev and X. Tata, Phys. Rev. D 90, 115007 (2014).

[24] C. Han, D. Kim, S. Munir and M. Park, JHEP 1504, 132 (2015).

[25] D. Barducci, et al., arXiv:1504.02472 [hep-ph].

[26] A. G. Delannoy, et al., Phys. Rev. Lett. 111, 061801 (2013).

[27] G. Aad et al. [ATLAS Collaboration], JHEP 1410, 24
(2014).

[28] S. Chatrchyan et al. [CMS Collaboration], Phys. Lett. B 725, 243 (2013).

[29] G. Aad et al. [ATLAS Collaboration], Phys. Rev. D 90, 052008 (2014); JHEP 1411, 118 (2014); Eur. Phys. J. C 74, 3109 (2014); JHEP 1409, 015 (2014); JHEP 1406, 124 (2014); JHEP 1310, 189 (2013).

[30] S. Chatrchyan et al. [CMS Collaboration], Phys. Rev. Lett. 112, 161802 (2014); Phys. Lett. B 736, 371 (2014); Phys. Lett. B 743, 503 (2015); Eur. Phys. J. C 73, 2677 (2013); arXiv:1503.08037 [hep-ex].

[31] Y. Bai, H. C. Cheng, J. Gallicchio and J. Gu, JHEP 1207, 110 (2012); JHEP 1308, 085 (2013).

[32] D. E. Kaplan, K. Rehermann and D. Stolarski, JHEP 1207, 119 (2012).

[33] T. Plehn, M. Spannowsky and M. Takeuchi, JHEP 1208 , 091 (2012); Phys. Rev. D 85, 034029 (2012); JHEP 1105, 135 (2011); T. Plehn, M. Spannowsky, M. Takeuchi and D. Zerwas, JHEP 1010, 078 (2010).

[34] J. Thaler and K. Van Tilburg, JHEP 1202, 093 (2012);

[35] Z. Han, A. Katz, D. Krohn and M. Reece, JHEP 1208, 083 (2012).

[36] M. Drees, M. Hanussek and J. S. Kim, Phys. Rev. D 86, 035024 (2012).

[37] D. S. M. Alves et al., Phys. Rev. D 87, 035016 (2013) [arXiv:1205.5805 [hep-ph]].

[38] X. -J. Bi, Q. -S. Yan and P. -F. Yin, Phys. Rev. D 85, 035005 (2012).

[39] J. Cao, et al., JHEP 1211, 039 (2012).

[40] M. L. Graesser and J. Shelton, Phys. Rev. Lett. 111, 121802 (2013).

[41] G. Ferretti, R. Franceschini, C. Petersson and R. Torre, arXiv:1502.01721 [hep-ph].

[42] M. Czakon, et al., Phys. Rev. Lett. 113, 201803 (2014).

[43] B. Fuks, P. Richardson and A. Wilcock, Eur. Phys. J. C 75, no. 7, 308 (2015).

[44] B. Nachman and C. G. Lester, Phys. Rev. D 88, 075013 (2013)

[45] T. Eifert and B. Nachman, Phys. Lett. B 743, 218 (2015).

[46] A. Ismail, R. Schwienhorst, J. S. Virzi and D. G. E. Walker, Phys. Rev. D 91, 074002 (2015).

[47] M. Papucci, J. T. Ruderman and A. Weiler, JHEP 1209, 035 (2012).

[48] L. J. Hall, D. Pinner and J. T. Ruderman, JHEP 1204, 131 (2012).

[49] O. Buchmueller and J. Marrouche, Int. J. Mod. Phys. A 29, 1450032 (2014).

[50] G. D. Kribs, A. Martin and A. Menon, Phys. Rev. D 88, 035025 (2013).

[51] C. Han, et al., JHEP 1310, 216 (2013).

[52] K. Kowalska and E. M. Sessolo, Phys. Rev. D 88, 075001 (2013).

[53] M. Cahill-Rowley, J. Hewett, A. Ismail and T. Rizzo, Phys. Rev. D 90, 095017 (2014).

[54] K. J. de Vries, et al., arXiv:1504.03260 [hep-ph].

[55] H. Abe, J. Kawamura and Y. Omura, arXiv:1505.03729 [hep-ph].

[56] B. Batell and S. Jung, arXiv:1504.01740 [hep-ph].

[57] A. Katz, M. Reece and A. Sajjad, JHEP 1410, 102 (2014) [arXiv:1406.1172 [hep-ph]].

[58] M. Drees and J. S. Kim, arXiv:1511.04461 [hep-ph];

[59] W. Beenakker et al., Nucl. Phys. B 515, 3 (1998).

[60] D. Goncalves, D. Lopez-Val, K. Mawatari and T. Plehn, Phys. Rev. D 90, 075007 (2014); D. Lopez-Val, et al., 
PoS LL 2012, 048 (2012); D. Goncalves, et al., Phys. Rev. D 87, 014002 (2013).

[61] J. Pumplin, et al., JHEP 0207, 012 (2002).

[62] B. C. Allanach, Comput. Phys. Commun. 143, 305 (2002).

[63] M. Muhlleitner, A. Djouadi and Y. Mambrini, Comput. Phys. Commun. 168, 46 (2005)

[64] J. Andrea, B. Fuks and F. Maltoni, Phys. Rev. D 84, 074025 (2011).

[65] J. L. Agram, J. Andrea, M. Buttignol, E. Conte and B. Fuks, Phys. Rev. D 89, no. 1, 014028 (2014).

[66] J. Wang, C. S. Li, D. Y. Shao and H. Zhang, Phys. Rev. D 86, 034008 (2012).

[67] Y. L. Dokshitzer, G. D. Leder, S. Moretti and B. R. Webber, JHEP 9708, 001 (1997).

[68] M. Cacciari, G. P. Salam and G. Soyez, Eur. Phys. J. C 72, 1896 (2012).
[69] M. Cacciari, G. P. Salam and G. Soyez, JHEP 0804, 063 (2008).

[70] T. Lin, E. W. Kolb and L. T. Wang, Phys. Rev. D 88, 063510 (2013).

[71] V. Khachatryan et al. [CMS Collaboration], Phys. Rev. Lett. 114, no. 10, 101801 (2015).

[72] J. Alwall et al., JHEP 1106, 128 (2011).

[73] F. Caravaglios, M. L. Mangano, M. Moretti and R. Pittau, Nucl. Phys. B 539, 215 (1999).

[74] https://cp3.irmp.ucl.ac.be/projects/madgraph/wiki/Matching

[75] T. Sjostrand, S. Mrenna and P. Z. Skands, JHEP 0605, 026 (2006).

[76] J. de Favereau, et al., arXiv:1307.6346 [hep-ex].

[77] CMS Collaboration, b-Jet Identification in the CMS Experiment, CMS-PAS-BTV-11-004.

[78] N. Kidonakis Phys. Rev. D84, 011504(2011). 